\title{
Energy Supply Chains Planning: Risk-based Optimization
}

\author{
Shiyu Chen \\ Energy Department, Politecnico di Milano, Italy. \\ E-mail: shiyu.chen@polimi.it \\ Wei Wang \\ Department of Mechanical Engineering, City University of Hong Kong, Hong Kong, China. \\ E-mail:wwang326@cityu.edu.hk \\ Enrico Zio \\ Energy Department, Politecnico di Milano, Italy. \\ MINES ParisTech, PSL Research University, CRC, Sophia Antipolis, France. \\ Eminent Scholar, Department of Nuclear Engineering, College of Engineering, Kyung Hee University, \\ Republic of Korea. \\ E-mail: enrico.zio@polimi.it
}

The planning of an Energy Supply Chain (ESC) aims at maximizing the benefits of the ESC agents, while satisfying the demands of the customers (Stadtler (2005); Shiyu et al. (2020)). Demand variability and supply disruption, originating from the connectivity between supply and demand, can disturb the agents interactions and impair the agents management (Govindan et al. (2017)). In this study, we propose a risk-based optimization approach for the management of ESC. We introduce a Conditional Value at Risk (CVaR) measure with the purpose of measuring and controlling the risk to the ESC management. A multi-objective optimization based by the Non-dominated Sorting Genetic Algorithm (NSGA-II) is performed to search for the solution optimal with respect to the maximization of the ESC total profit and the minimization of the risk under uncertainties. For demonstration, an application is carried out considering a specific oil\&gas ESC model with five layers, including crude oil producers, storages, refineries, terminal storages and retailers. Results show that the optimization approach enables the trade-off between the ESC optimal planning and the source of risk that it is subjected to.

Keywords: Energy Supply Chain (ESC), Agent-based Modeling (ABM), risk-based optimization, CVaR, NSGA-II, uncertainty.

\section{Introduction}

An Energy Supply Chain (ESC) is made up of a number of agents interacting with each other. The agents in the ESC, such as crude oil producers, refineries, storages are physically and functionally heterogeneous, and organized in a hierarchy such that what happens to one agent can easily spread through the ESC and affect other agents (Zio (2016)). In such environment, agents are required to maximize the benefits, while satisfying the demands of the customers(Stadtler (2005); Shiyu et al. (2020)). Therefore, modeling and optimizing of ESCs become important for its planning.

This paper studies the planning problem of ESC under uncertainties and risks. Demand, production and supply uncertainties, related to product characteristics, are the major sources of uncertainties (Ho et al. (2005)). Besides these uncertainties, consideration must be given also to supply chain risks of two types: disruption risk and operational risk (Kleindorfer and Saad (2005); Tang (2006)). The disruption risks are related to circumstances such as natural disaster, terrorist attacks and labor strikes, whereas operation risks are caused by high uncertainty and unbalance between supply and demand (Lockamy III and McCormack (2010); Sreedevi and Saranga (2017)). These risks influence the ESC function (Heckmann et al. (2015)).

To address this problem, we propose a simulation-based optimization method which consists of a simulation module and an optimization module. For the simulation former, we rely on Agent-based Modeling (ABM), which is a powerful technique for modelling large interconnected systems (Bonabeau (2002)). The oil companies, factories, producers, customers in the ESC are functionally described as agents who can interact with each other(Farmer and Foley (2009)).

In this ESC model, the demand and supply 
uncertainties, and the disruption risks are considered. Demand and supply uncertainties change the plant operations and consequently the production planing. To consider these problems, we correspondingly define the objective functions.

For the multi-objective optimization module, the objective functions are the total profit and the Conditional Value at Risk (CVaR). On one hand, the profit is a most crucial factor for oil companies. On the other hand, we consider CVaR to measure the "tail risk" of an investment portfolio, which is widely used in supply chain portfolio optimization. For example, Carneiro et al. (2010) uses CVaR as a risk measure constraint to maximize returns with risk constraints. Soleimani and Govindan (2014) applies CVaR as a risk evaluator to study design and planning of a reverse supply chain network in a two-stage stochastic programming framework. Qiu et al. (2014) studies a robust inventory decision problem by applying the CVaR risk measure under discrete demand distribution uncertainty. Thus, it can be seen that $\mathrm{CVaR}$ is an effective tool to manage risk. Hence, we apply CVaR to measure the disruption risk in the ESC. Finally, the multi-objective problem defined is solved by applying a simulation-based genetic algorithm optimization method.

The rest of the paper is organized as follows. In Section 2, we present the Agent-Based ESC model. Section 3 introduces the ABM-MOO formulation and solution framework. Section 4 illustrates the case study and the results of the ESC's profit and $\mathrm{CVaR}$ optimization under disruption risk and uncertainties. A discussion is given in Section 5.

\section{The ESC Model}

The ESC is described by ABM, which considers the logical rules of the agents behavior and interactions (Macal and North (2005)). The model allows simulating the ESC transaction processes in an uncertain, dynamic and time-dependent environment (Labarthe et al. (2007); Julka et al. (2002); Parunak et al. (1998); Farmer and Foley (2009)). In our model, we take into account the demand and supply uncertainties, and the disruption risk.

\subsection{Modeling of Agents Uncertain Behavior}

We consider an ESC with $L$ layers and each $l$-th layer consists of $V_{l}$ agents, $a_{l, 1}, a_{l, 2}, \ldots, a_{l, v}, \ldots, a_{l, V_{l}}$ (Figure 1). In the ESC, the orders sent from the demanders of Layer 1 flow layer-by-layer to the end layer $L$, whose $V_{L}$ agents are suppliers that send the supply backward to the demanders.

An agent $a_{l, v}, v=1,2, \ldots, V_{l}$, in the $l$-th layer, is assigned with behaviors, which allow the agent to adaptively interact with the others. The details of the model are described in the following.

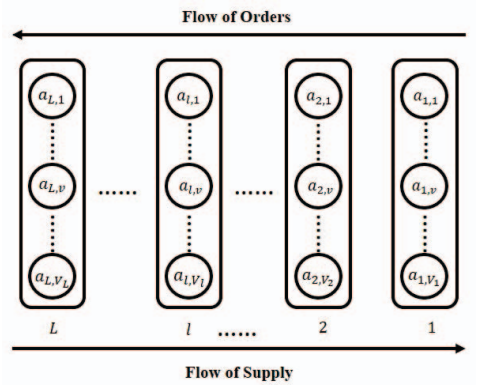

Fig. 1. The ABM-ESC model

\subsubsection{Sending Orders}

The process of sending orders of an agent $a_{l, v}$ in the $l$-th layer (Fig.2) is as follows:

(a) $a_{l, v}$ chooses the supplier $a_{l+1, v^{\prime}}$ in the upper layer $l+1$, based on capacity and cost of satisfying the order.

(b) $a_{l, v}$ sends orders to $a_{l+1, v^{\prime}}$.

(c) $a_{l, v}$ updates the list of alternative suppliers, taking into account the capacity of supply.

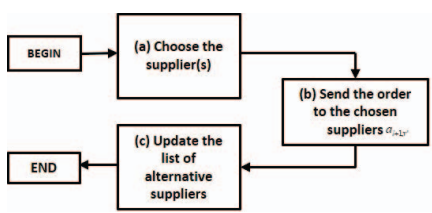

Fig. 2. The process of sending orders

\subsubsection{Receiving Orders and Choosing Demanders}

The process of an agent $a_{l, v}$ receiving orders and choosing demander(s) is shown in Fig.3:

(a) $a_{l, v}$ receives the order from $a_{l-1, v^{\prime \prime}}$ in the lower layer $l-1$.

(b) $a_{l, v}$ checks whether the received order is empty:

- If yes, $a_{l, v}$ does not choose demanders.

- Otherwise, (c) $a_{l, v}$ checks whether it has available productions that satisfy the received orders.

- If yes, (d) $a_{l, v}$ checks whether the received orders exceed the existing production limitation $U_{l, v}(t)$ defined as:

$$
U_{l, v}(t)=S_{l, v}(t)-S_{l, v}^{*}(t)
$$

where $S_{l, v}$ is the storage of $a_{l, v}$ and $S_{l, v}^{*}$ is the back-up safety storage of $a_{l, v}$. 
* If yes, (e) $a_{l, v}$ refuses the order from $a_{l-1, v^{\prime \prime}}$ demanded with the lowest bid price, and returns to $(\mathrm{d})$.

* Otherwise, (f) the agent $a_{l, v}$ accepts the order and makes a contract with $a_{l-1, v^{\prime \prime}}$. Then, the existing oil production limitation of the agent $a_{l, v}$ (Eq.(2)) updates for the next time $t+1$ :

$$
U_{l, v}(t+1)=U_{l, v}(t)-\sum_{v^{\prime \prime}, v^{\prime \prime} \in\left\{v_{a}\right\}} x_{l, v}^{l-1, v^{\prime \prime}}(t)
$$

where, $x_{l, v}^{l-1, v^{\prime \prime}}(t)$ is the amount of orders accepted by the agent $a_{l, v}$ which are sent by the agent $a_{l-1, v^{\prime \prime}}$

- Otherwise, (g) $a_{l, v}$ sends a response back to the demander $a_{l-1, v^{\prime \prime}}$.

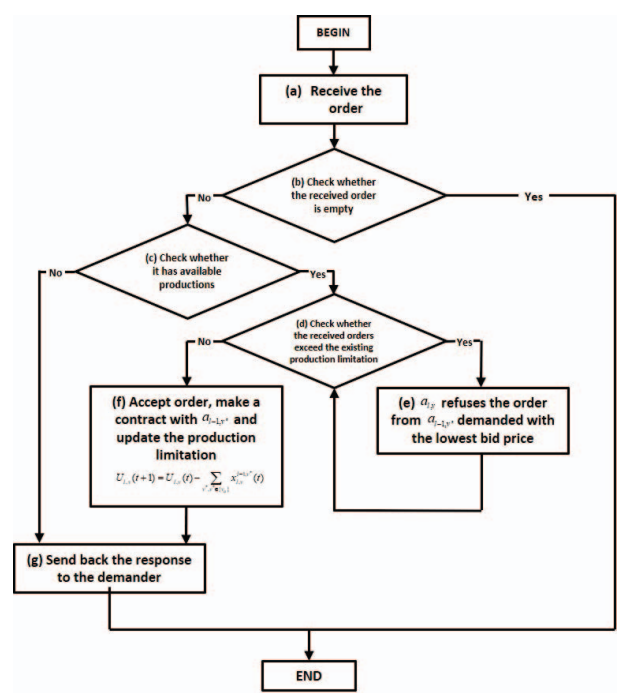

Fig. 3. The process of receiving orders and choosing deman$\operatorname{der}(\mathrm{s})$

\subsubsection{Response}

One demander $a_{l, v}$ may negotiate with its supplier $a_{l+1, v^{\prime}}$, in case of receiving a response from $a_{l+1, v^{\prime}}$. This process is shown in Fig.4 and described as follows:

(a) The agent $a_{l, v}$ receives a response from the supplier $a_{l+1, v^{\prime}}$

(b) Check whether the order plan is satisfied:

- If yes, $a_{l, v}$ stops sending the order plan.

- Otherwise, (c) $a_{l, v}$ checks whether all the alternative suppliers have been considered:
- If yes, the agent $a_{l, v}$ stops sending the order plan.

- Otherwise, (d) the agent $a_{l, v}$ updates its demands,

$$
y_{l, v}^{l+1, v^{\prime}}(t+1)=y_{l, v}^{l+1, v^{\prime}}(t)-\sum_{v^{\prime}, v^{\prime} \in\left\{v_{a}\right\}} x_{l+1, v^{\prime}}^{l, v}(t)
$$

where, $y_{l, v}^{l+1, v^{\prime}}(t)$ is the amount of orders sent by the agent $a_{l, v}$ which are received by the agent $a_{l+1, v^{\prime}}$ at time $t, x_{l+1, v^{\prime}}^{l, v}(t)$ is the amount of orders accepted by the agent $a_{l+1, v^{\prime}}$ which are sent by the agent $a_{l, v}$ at time $t$.

And, (e) send orders (as discussed in Section 2.1.1) again.

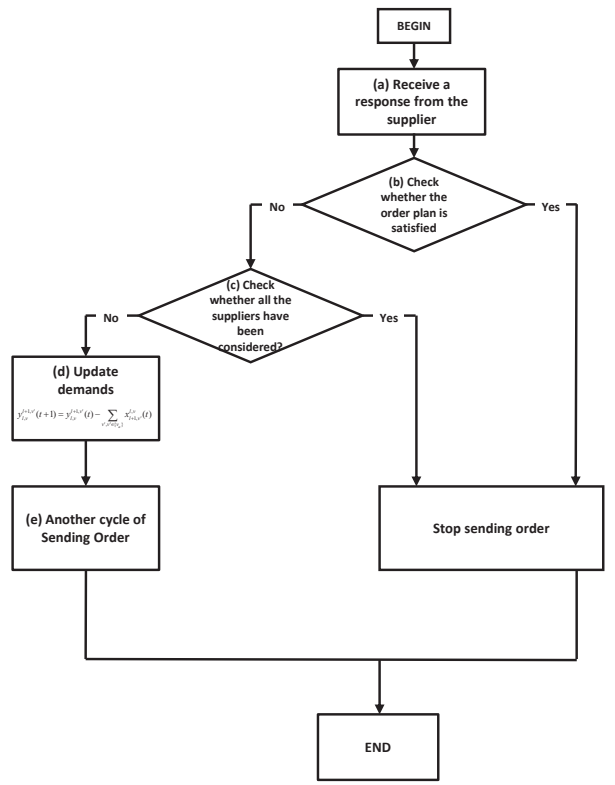

Fig. 4. The process of response

\subsubsection{Selling Production}

An agent $a_{l, v}$ should sell productions after accepting an order plan. This process is shown in Fig.5 and defined as follows:

(a) $a_{l, v}$ checks whether it stores enough productions satisfying the accepted order plan:

- If yes, (b) sells the productions to the demander $a_{l-1, v^{\prime \prime}}$ and updates the set of accepted orders and the storage for the next time $t+1$ (Eq.(4)): 


$$
S_{l, v}(t+1)=S_{l, v}(t)-\sum_{v^{\prime \prime}, v^{\prime \prime} \in\left\{v_{a}\right\}} z_{l, v}^{l-1, v^{\prime \prime}}(t)
$$

where $S_{l, v}$ is the production storage of $a_{l, v}$ and, $z_{l, v}^{l-1, v^{\prime \prime}}(t)$ is the amount of the production sold by $a_{l, v}$ to $a_{l-1, v^{\prime \prime}}$.

- Otherwise, (c) $a_{l, v}$ sells the productions to the demander who makes its income highest, and, then, updates the set of accepted orders and the storage $S_{l, v}(t+1)$.

(d) Check whether the storage or the set of accepted orders is empty.

- If not, repeat (c).

- Otherwise, end.

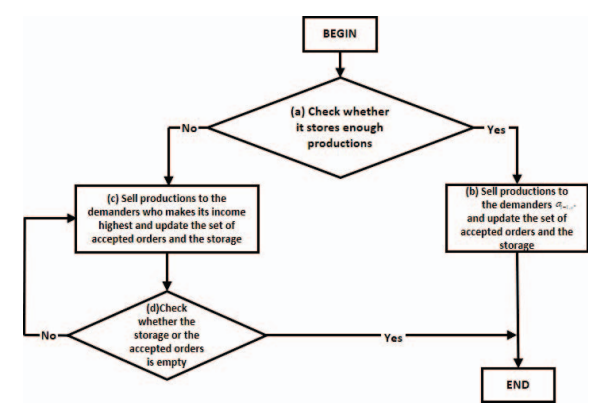

Fig. 5. The process of selling production

\subsubsection{Receiving Production}

An agent $a_{l, v}$ receives the oil production from the upstream agents $a_{l+1, v^{\prime}}$ and updates its storage $S_{l, v}(t+1)$ for the next time $t+1$ :

$$
S_{l, v}(t+1)=S_{l, v}(t)+w_{l, v}^{l+1, v^{\prime}}(t) \cdot k_{l, v}
$$

where $w_{l, v}^{l+1, v^{\prime}}(t)$ is the amount of the oil production sent by the the agent $a_{l, v}$ which are received by the agent $a_{l+1, v^{\prime}}$ time $\mathrm{t}, k_{l, v}$ is the production capacity of $a_{l, v}$,

\subsection{Uncertainty and risk assessment}

In this study, we use the Conditional Value-at-Risk ( $\mathrm{CVaR}$ ) to measure the risk in the cost of supply (Carneiro et al. (2010); Qiu et al. (2014); Gebreslassie et al. (2012); Zhang and Zhang (2019); Saghaei et al. (2020); Snoeck et al. (2019)). The definitions of VaR and CVaR are shown as follows:

$$
\operatorname{VaR}_{\alpha}(X)=\inf \left\{Z \in R: F_{X}(Z)>\alpha\right\}
$$

where $X$ is the loss, $F_{X}$ is the discrete approximation of the probability distribution of the loss $X$, $\alpha$ is the $\alpha$-percentile for the function $F_{X}, Z$ is the smallest value of loss whose probability is greater than $\alpha$.

$$
C \operatorname{VaR}_{\alpha}(X)=E\left(X \mid X \geq \operatorname{VaR}_{\alpha}(X)\right)
$$

where $X$ is the loss and $E(X)$ is the expected value of $X$ which is larger than $\operatorname{VaR}_{\alpha}(X)$ defined in 6 .

In order to understand the CVaR in a comprehensive view, we draw a graphical definition in Fig.6.

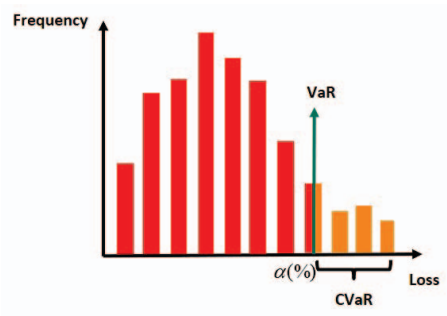

Fig. 6. Distribution of sending production loss

Fig.6 shows that VaR is the smallest value of the loss for a confidence level $\alpha$ of the probability distribution. Although the VaR has been widely used in measuring risk, it also presents some deficiencies (Carneiro et al. (2010)).

$\mathrm{CVaR}$ is the expected value of the loss given that the loss is greater than or equal to the $\operatorname{VaR}_{\alpha}(X)$. The definitions and properties of $\mathrm{CVaR}$ are given in detail in ( citerockafellar2006generalized,rockafellar2002conditional).

The ESC planning problem under risk and uncertainty is described in Section 2.3.

\section{3. $\mathrm{MOO}$ problem formulation}

In the ESC, every agent wants to maximize its profit. Here, we assume that the main income is from selling oil production and the main cost is in buying oil production.

Eq.(8) defines the ESC total profit $P$ over a time horizon $T$ :

$$
P=\sum_{t=1}^{T} \sum_{l=1}^{L} \sum_{v=1}^{V_{l}}(A-B-C-D)
$$

$A$ is related to the income from selling the oil production to the customer, expressed as:

$$
A=\sum_{v^{\prime \prime}=1}^{V_{l-1}^{\prime \prime}} p_{l, v}^{l-1, v^{\prime \prime}} z_{l, v}^{l-1, v^{\prime \prime}}(t)
$$


where $p_{l, v}^{l-1, v^{\prime \prime}}$ is the unit price of the agent $a_{l, v}$ when selling the oil production to the agent $a_{l-1, v^{\prime \prime}}, z_{l, v}^{l-1, v^{\prime \prime}}(t)$ is the production sent by the agent $a_{l, v}$, which is received by the agent $a_{l-1, v^{\prime \prime}}$ at time $t$.

$B$ is the purchase cost, which includes the procurement cost plus other costs like the transportation cost, the labor cost and so forth:

$$
B=\sum_{v^{\prime}=1}^{V^{\prime}}\left(p_{l+1, v^{\prime}}^{l, v}+o_{l, v}^{l+1, v^{\prime}}\right) w_{l, v,}^{l+1, v^{\prime}}(t)
$$

where $p_{l+1, v^{\prime}}^{l, v}$ is the unit price of the agent $a_{l+1, v^{\prime}}$ for selling the oil production to agent $a_{l, v}, o_{l, v}^{l+1, v^{\prime}}$ is the unit price for the other costs, $w_{l, v, t}^{l+1, v^{\prime}}$ is the amount of the oil production sent by the agent $a_{l+1, v^{\prime}}$ and which is received by the agent $a_{l, v}$.

The item $C$ accounts for the storage cost:

$$
C=c_{l, v}^{S} S_{l, v}(t)
$$

where $c_{l, v}^{S}$ is the agent $a_{l, v}$ storage unit cost, $S_{l, v, t}$ is the production storage of the agent $a_{l, v}$ at time $t$.

Finally, $D$ is the penalty for supply shortage:

$$
D=\alpha_{l, v} \sum_{v^{\prime \prime}=1}^{V_{l-1}^{\prime \prime}}\left(x_{l, v}^{l-1, v^{\prime \prime}}(t)-z_{l, v}^{l-1, v^{\prime \prime}}(t)\right)
$$

where $\alpha_{l, v}$ is the unit penalty cost, $x_{l, v}^{l-, v^{\prime \prime}}(t)$ is the amount of orders accepted by the agent $a_{l, v}$ which are sent by the agent, $z_{l, v}^{l-1, v^{\prime \prime}}(t)$ is the amount of oil production sent by the agent $a_{l, v}$, which are received by the agent $a_{l-1, v^{\prime \prime}}$ at time $t$.

Then, the total cost at time $t$ could be defined as:

$$
E(t)=\sum_{l=1}^{L} \sum_{v=1}^{V_{l}}(B+C+D)
$$

The general MOO problem is, then, formulated as:

$$
\begin{gathered}
\max P\left(\bar{y}_{1,1}, \ldots, \bar{y}_{l, v}, \ldots, \bar{y}_{L-1, V_{L}}, p_{2,1}^{1,1}, \ldots,\right. \\
\left.p_{l, v}^{l-1, v^{\prime \prime}}, \ldots, p_{L, V_{L}}^{L-1, V_{L-1}}\right)
\end{gathered}
$$

$$
\begin{aligned}
\min C \operatorname{VaR}\left(\bar{y}_{1,1}, \ldots, \bar{y}_{l, v}, \ldots, \bar{y}_{L-1, V_{L}},\right. \\
\left.p_{2,1}^{1,1}, \ldots, p_{l, v}^{l-1, v^{\prime \prime}}, \ldots, p_{L, V_{L}}^{L-1, V_{L-1}}\right) \\
=\operatorname{CVaR} R_{\alpha}\left(\sum_{l=1}^{L} \sum_{v=1}^{V_{l}} E_{l, v}(t)\right)
\end{aligned}
$$

s.t.

$$
\bar{y}_{l}^{\min } \leq \bar{y}_{l, v, t} \leq \bar{y}_{l}^{\max }
$$

$$
p_{l}^{\min } \leq p_{l, v}^{l-1, v^{\prime \prime}} \leq p_{l}^{\max }
$$

Eq.14 maximizes the total profit and Eq.15 minimizes the risk, simultaneously. The constraint 16 and the constraint 17 give the feasible region for the average orders and the price.

\section{The ABM-MOO Framework}

GA is used to search a set of feasible solutions optimal with respect to the objective functions. These solutions (chromosomes, in GA terminology) are input in the simulation model. Fig.7 shows the framework of the hybrid simulationbased optimization process.

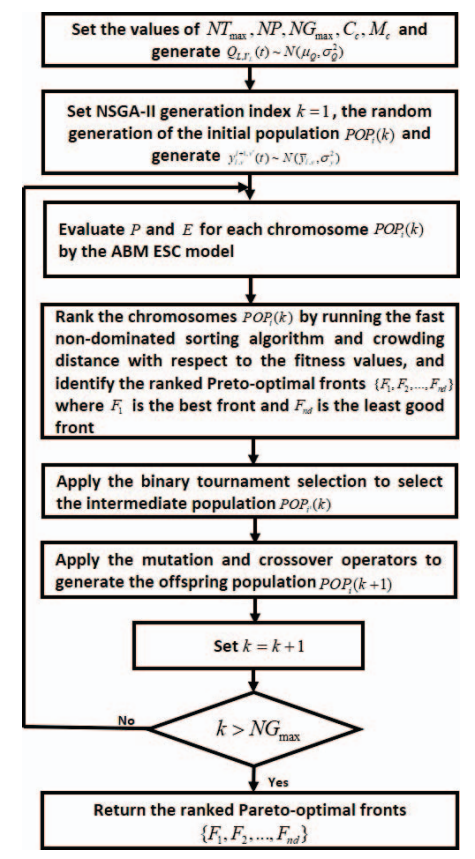

Fig. 7. The flowchart of the ABM-MOO framework

A set of Pareto solutions is, eventually, identified and a best-compromised solution can be chosen among them by the Min-Max method (Marseguerra et al. (2005); Belegundu and Chandrupatla (2019)). Given the Pareto solutions $H \equiv$ $(P, C V a R)$ the relative deviations of $P$ and $C V a R$ are defined, respectively:

$$
z_{P}=\frac{\left|P-P^{\min }\right|}{P^{\max }-P^{\min }}
$$

where $P^{\min }$ and $P^{\max }$ are the minimum and maximum of the fitness values $P$, respectively. 


$$
z_{C V a R}=\frac{\left|C V a R-C V a R^{\min }\right|}{C V a R^{\max }-C V a R^{\min }}
$$

where $C V a R^{\min }$ and $C V a R^{\max }$ are the minimum and maximum of the fitness values $C V a R$, respectively.

The best-compromised solution is determined as

$$
z_{H}=\min \left[\max \left\{z_{P}, z_{C V a R}\right\}\right]
$$

\section{Case Study}

An ESC ABM of five layers is considered. This includes retailers (Layer1), terminal storages (Layer2), refineries (Layer3), storages (Layer4) and crude oil producers (Layer5). In Layer1, Layer2 and Layer3, there are 3 agents respectively. In Layer4 and Layer5, there are 2 agents in each layer. In this ESC, we assume that there is $10 \%$ probability that the refinery 3 is disrupted. When it is disrupted, it is not able to send oil production to down stream demanders.

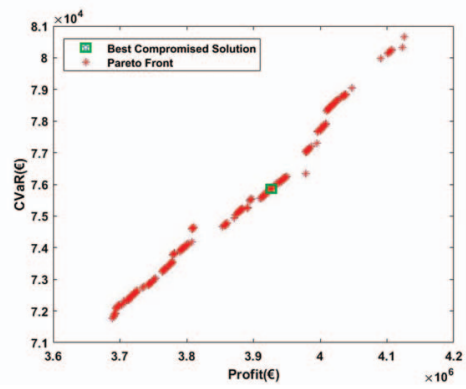

Fig. 8. The Pareto front with the best-compromised solution

Fig. 8 shows the Pareto front which reflects that the CVaR increases when the ESC total profit tends to be large. Then increasing $\mathrm{CVaR}$ means increasing risk: if the agents want to get more profit they have to face larger risk which makes sense because high profit are always accompanied by high risk. According to Eq.(20) discussed in Section 3, the best compromised solution is identified, and the total profit $P$ equals to $€ 3924956.04$ and $C V a R$ equals to $€ 75851.93$.

Fig.9 shows the cost frequency distribution in the normal state which is optimized in the literature (Shiyu et al. (2020)). Fig.10 shows the cost frequency distribution with disruption risks if we do not take any measure to control them. Fig.11 shows the cost frequency distribution with disruption risk if the ESC is optimized.

Fig.9 shows that if the ESC is without disruption risk, the cost is distributed in the range from

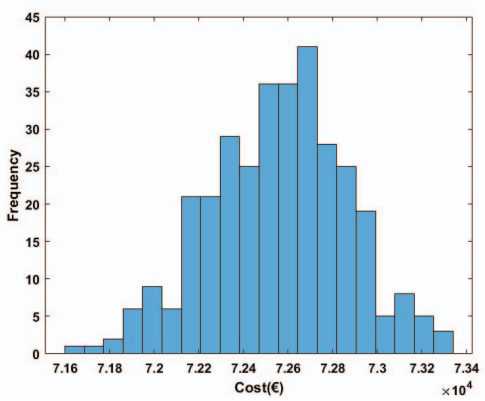

Fig. 9. The cost frequency distribution under the ESC normal state

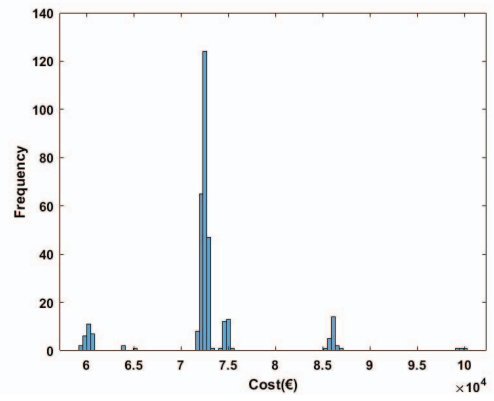

Fig. 10. The cost frequency distribution with the ESC disruption risk

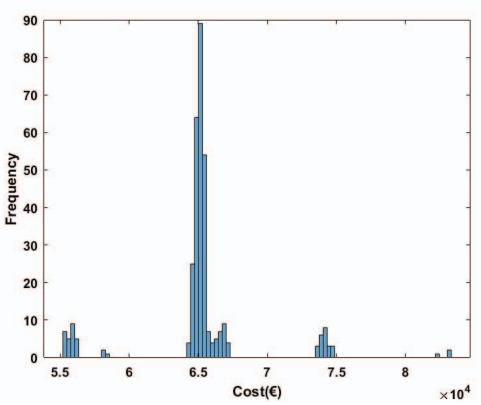

Fig. 11. The cost frequency distribution with the ESC disruption risk after optimization

$€ 7.16 \times 10^{4}$ to $€ 7.34 \times 10^{4}$ which is a relatively concentrated range. Comparing Fig. 9 with Fig.10 and Fig.11, the range of the cost distribution increases, because the disruption risks cause the refinery storage cost and shortage penalty to increase. On the other hand, after the disruption happens, some oil production has to be stored in Refinery 3. When the ESC goes back to normal, Refinery 3 does not need to order new oil produc- 
tion from up stream storage, so the procurement cost is decreasing, which further increases the range of the cost distribution. In Fig. 10, the $95 \%$ confidence level on the right-hand of the cost is $€ 88650$. In Fig. 11, the 95\% confidence level on the right-hand of the cost is $€ 75851.93$. Comparing Fig. 10 with Fig.11, the proposed ABM-MOO framework is effective to decrease the $\mathrm{CVaR}$ related to the risk disruption.

\section{Conclusions}

The objective of this study is to manage the production planning problem in Energy Supply Chains (ESCs), where the agent interaction behavior is uncertain and the ESC faces disruption risk. In order to address this problem, we propose a simulation-based multi-objective optimization framework which enables decision making on planning production, including the price and the amount of purchased oil production. Firstly, we use Agent-based Modeling (ABM) to model and simulate the agent behaviors and the ESC transaction processes. Secondly, we use Nondominated Sorting Genetic Algorithm (NSGA-II) to get the optimal solutions that maximize the ESC total profit and minimize the disruption risk. An oil ESC model with five layers, including crude oil producers, storages, refineries, terminal storages and retailers is presented to demonstrate the methodology.

For future research, we think that the cooperative and competitive relationships among agents can be considered in the ESC optimization problem.

\section{Acknowledge}

This work is supported by the scholarship from China Scholarship Council (CSC).

\section{Appendix A. Nomenclature}

$\left\{v_{a}\right\}$ : The set of the agent indexes whose orders are accepted.

$a_{l, v}$ : The $v$-th agent in the layer $l$.

$k_{l, v}$ : The production capacity of agent $a_{l, v}$.

$\alpha_{l, v}$ : The unit penalty cost.

$\varepsilon_{P}:$ An arbitrary large number for total profit.

$\varepsilon_{E}:$ An arbitrary large number for uncertainty.

$p_{l}^{\min }$ : The minimum for the unit price.

$p_{l}^{\max }$ : The maximum for the unit price.

$\bar{y}_{l}^{\min }$ : The minimum for the average orders.

$\bar{y}_{l}^{\max }$ : The maximum for the average orders.

$r(t)$ : The number of agents who get a loss after the oil production transaction.

$Q_{L, V_{L}}(t)$ : The amount of productions, which are produced by the agents in the last layer at time $t$.

$U_{l, v}(t)$ : The residual oil production limitation of the agent $a_{l, v}$ at time $t$.

$S_{l, v}(t)$ : The storage of the agent $a_{l, v}$ at time $t$.
$S_{l, v}^{*}(t)$ : The back up safety storage in the agent $a_{l, v}$ at time $t$.

$x_{l, v}^{l-, v^{\prime \prime}}(t)$ : The amount of orders accepted by the agent $a_{l, v}$, which are sent by the agent $a_{l-1, v^{\prime \prime}}$ at time $t$.

$y_{l, v}^{l+1, v^{\prime}}(t)$ : The amount of orders sent by the agent $a_{l, v}$, which are received by the agent $a_{l+1, v^{\prime}}$ at time $t$.

$z_{l, v}^{l-1, v^{\prime \prime}}(t)$ : The amount of the oil production sent by the agent $a_{l, v}$, which are received by the agent $a_{l-1, v^{\prime \prime}}$ at time $t$.

$w_{l, v}^{l+1, v^{\prime}}(t)$ : The amount of the oil production sent by the the agent $a_{l+1, v^{\prime}}$, which are received by the agent $a_{l, v}$ at time $t$.

$o_{l, v}^{l+1, v^{\prime}}:$ The unit price for the other cost.

$p_{l, v}^{l-1, v^{\prime \prime}}:$ The unit price of the agent $a_{l, v}$ by selling the oil production to the agent $a_{l-1, v^{\prime \prime}}$.

$c_{l, v}^{S}$ : The agent $a_{l, v}$ storage unit cost.

$\mu_{l}$ : The mean of agents profits in the layer $l$.

$\sigma_{l}$ : The standard deviation of agents profits in the layer $l$.

$\mu_{Q}$ : The mean of agents production amount $Q_{L, V_{L}}(t)$.

$\sigma_{Q}$ : The standard deviation of agents production amount $Q_{L, V_{L}}(t)$.

$\bar{y}_{l, v}$ : The amount of average orders sent by the agent $a_{l, v}$ at time $t$.

$\sigma_{y}$ : The standard deviation of agents orders

$y_{l, v}^{l+1, v^{\prime}}(t)$.

$N T_{\max }$ : The total transaction time.

$N G_{\max }$ : Maximum number of GA generations.

$N P$ : GA population size.

$C_{c}$ : Crossover coefficient.

$M_{c}$ : Mutation coefficient.

\section{References}

Belegundu, A. D. and T. R. Chandrupatla (2019). Optimization concepts and applications in engineering. Cambridge University Press.

Bonabeau, E. (2002). Agent-based modeling: Methods and techniques for simulating human systems. Proceedings of the national academy of sciences 99(suppl 3), 7280-7287.

Carneiro, M. C., G. P. Ribas, and S. Hamacher (2010). Risk management in the oil supply chain: a cvar approach. Industrial \& Engineering Chemistry Research 49(7), 3286-3294.

Farmer, J. D. and D. Foley (2009). The economy needs agent-based modelling. $\mathrm{Na}$ ture 460(7256), 685-686.

Gebreslassie, B. H., Y. Yao, and F. You (2012). Design under uncertainty of hydrocarbon biorefinery supply chains: multiobjective stochastic programming models, decomposition algorith- 
$\mathrm{m}$, and a comparison between cvar and downside risk. AIChE Journal 58(7), 2155-2179.

Govindan, K., M. Fattahi, and E. Keyvanshokooh (2017). Supply chain network design under uncertainty: A comprehensive review and future research directions. European Journal of Operational Research 263(1), 108-141.

Heckmann, I., T. Comes, and S. Nickel (2015). A critical review on supply chain risk-definition, measure and modeling. Omega 52, 119-132.

Ho, C.-F., Y.-M. Tai, Y.-M. Tai, and Y.-P. Chi (2005). A structural approach to measuring uncertainty in supply chains. International Journal of Electronic Commerce 9(3), 91-114.

Julka, N., R. Srinivasan, and I. Karimi (2002). Agent-based supply chain managementł1: framework. Computers \& Chemical Engineering 26(12), 1755-1769.

Kleindorfer, P. R. and G. H. Saad (2005). Managing disruption risks in supply chains. Production and operations management 14(1), 53-68.

Labarthe, O., B. Espinasse, A. Ferrarini, and B. Montreuil (2007). Toward a methodological framework for agent-based modelling and simulation of supply chains in a mass customization context. Simulation Modelling Practice and Theory 15(2), 113-136.

Lockamy III, A. and K. McCormack (2010). Analysing risks in supply networks to facilitate outsourcing decisions. International Journal of Production Research 48(2), 593-611.

Macal, C. M. and M. J. North (2005). Tutorial on agent-based modeling and simulation. In Proceedings of the Winter Simulation Conference, 2005., pp. 14-pp. IEEE.

Marseguerra, M., E. Zio, and L. Podofillini (2005). Multiobjective spare part allocation by means of genetic algorithms and monte carlo simulation. Reliability Engineering \& System Safety 87(3), 325-335.

Parunak, H. V. D., R. Savit, and R. L. Riolo (1998). Agent-based modeling vs. equationbased modeling: A case study and users guide. In International Workshop on Multi-Agent Systems and Agent-Based Simulation, pp. 10-25. Springer.

Qiu, R., J. Shang, and X. Huang (2014). Robust inventory decision under distribution uncertainty: A cvar-based optimization approach. International Journal of Production Economics 153, 13-23.

Saghaei, M., H. Ghaderi, and H. Soleimani (2020). Design and optimization of biomass electricity supply chain with uncertainty in material quality, availability and market demand. Energy 197, 117165.

Shiyu, C., W. Wei, and Z. Enrico (2020). A simulation-based multi-objective optimization framework for the production planning problem in energy supply chains. In Progress $X X(\mathrm{XX})$, $\mathrm{XX}$.
Snoeck, A., M. Udenio, and J. C. Fransoo (2019). A stochastic program to evaluate disruption mitigation investments in the supply chain. European Journal of Operational Research 274(2), 516-530.

Soleimani, H. and K. Govindan (2014). Reverse logistics network design and planning utilizing conditional value at risk. European Journal of Operational Research 237(2), 487-497.

Sreedevi, R. and H. Saranga (2017). Uncertainty and supply chain risk: The moderating role of supply chain flexibility in risk mitigation. International Journal of Production Economics 193, 332-342.

Stadtler, H. (2005). Supply chain management and advanced planning--basics, overview and challenges. European journal of operational research 163(3), 575-588.

Tang, C. S. (2006). Perspectives in supply chain risk management. International journal of production economics 103(2), 451-488.

Zhang, G. and Q. Zhang (2019). Multiportfolio optimization with cvar risk measure. Journal of Data, Information and Management 1(3-4), 91-106.

Zio, E. (2016). Critical infrastructures vulnerability and risk analysis. European Journal for Security Research 1(2), 97-114. 